\title{
Autoimmune Studies and HLA Associations in SLE Patients from Mumbai
}

\author{
V. D. Pradhan, J.P. Devaraj, U. Shankarkumar and S. S. Badakere \\ Department of Autoimmune Disorders, Institute of Immunohaematology, \\ $13^{\text {th }}$ floor, K.E.M.Hospital, Parel, Mumbai 400 012, Maharastra, India \\ Fax: 91-22-4138521, Telephone: 91-22-4138518
}

KEYWORDS SLE; autoimmune antibodies; HLA; organ involvement

\begin{abstract}
To determine the autoimmune status and HLA allele distribution in SLE patients confirmed on serological and clinical grounds and compared with normal age matched ethnic control population from Mumbai. HLA A, B serological typing were carried out in 53 unrelated SLE patients and 110 healthy controls. Autoantibodies were detected by indirect immunofluorescence and ELISA techniques. The autoantibodies directed towards ANF, anti Sm, AHA were increased among the major organ involved patients while the antibodies like anti - dsDNA, anti - ssDNA, anti - nRNP, anti - SSA (Ro), anti - SSA (La) and RF were increased among the other patients with minor organ involvement. HLA A1, A2, and B27 alleles were positively associated, while HLA A19, and B15 alleles were negatively associated among the total SLE patients when compared to normal controls. It was further interesting to note that among the major organ involved severe patients A3, A28, and B7 where increased while A11, A19 and B17 were decreased. Among the minor organ involved patients it was A1, A2, A26, B5, B40 and B56 which were increased while A11, A28 and B7 were decreased. A significant twofold increase in the odds ratio for HLA A3, A28 and B27 alleles with increased Ro, La, Sm and ds DNA autoantibodies was observed when compared to controls. A significant twofold decrease in the odds ratio for HLA A19, A11, B7 and B15 alleles with decreased Ro, La, Sm and ds DNA autoantibodies was observed, when compared to normal controls suggesting the positive and negative associations of these HLA alleles among the SLE patients from Mumbai.
\end{abstract}

\section{INTRODUCTION}

SLE is an autoimmune disease characterized by multiple inflammation and production of autoantibodies to a range of intracellular antigens involved in gene transcription and translation. Genetic factors are likely to be important in both determining the overall susceptibility to SLE and in influencing remarkable clinical heterogeneity in disease expression observed between affected individuals (Eroglu and Kohler 2000). The more common clinical features seen in SLE patients include, skin and joint involvement's, renal involvement, neuropsychiatric complications and also some hematological abnormalities. Genetic factors along with the environmental factors strongly influence the development of SLE. Previous studies both in humans and animal models suggest that SLE is a complex disease with contribution from multiple genes. Multiple loci within the MHC have been implicated in susceptibility like HLA class II alleles, complement components and TNF loci.

The demonstration of Major Histocompatibility Complex (MHC) linked immune response (Ir.) and /or immune suppre-ssive (Is) genes
(Benacerraf 1981) has resulted in various disease association studies at population level to seek an association with MHC products, the human leukocyte antigens (HLA) (Tiwari and Terasaki 1985). Currently it is believed that some HLA alleles are in genetic linkage disequilibrium with certain disease related genes and they regulate the immune responses. The association of HLA haplotypes in autoimmune diseases supports this hypothesis. In all the autoimmune diseases the tendency for disease condition to run in the family is well documented Bale et al 1980; Shoenfeld and Isenberg 1989) and the association of HLA B27 with Ankylosing Spondylitis (AS) is consistent with a dominant or additive mode of inheritance on the same chromosome that predicts a predisposition to certain diseases. Since 1969, when the first case of Systemic Lupus Erythematosus (SLE) was reported from India, the disease has been extensively studied in different regions of the country viz, Chennai, Calcutta, Mumbai and New Delhi. A statistically significant clinical correlation comparing the clinical parameters from other racial groups of the world have been reported in Indian SLE patients (Malaviya et al. 1997). HLA association 
studies from Indian SLE patients are very much limited (Mehra et al. 1993) and further varying interethnic differences in the associations have been reported from UK, South African and Iceland populations (Steinsson et al. 1998).

\section{MATERIALS AND METHODS}

\section{Patients and Controls}

The SLE patients exhibiting the clinical manifestations as described by 1982 revised ARA diagnostic criteria (Tan et al 1982; Alarcon-Segoria 1984) were studied for their HLA antigen distribution and autoimmune parameters. The patients were referred during 1996-1999. A detailed questionnaire regarding the patient history, pathological investigations, drug regimen, clinical details and other population specific details were recorded. The average age group of the patients were $08 \pm 2$ to $50 \pm 2$ with a 1: 9 male to female ratio.

\section{Clinical Information}

The clinical presentation of these cases has been summarized in Table 1. Major Organ involvement patients selection criteria based on (i) Kidneys with gross proteinuria $>500 \mathrm{mg} / 24$ hour, serum creatinine $>2 \mathrm{mg} / \mathrm{dl}$, Grade III to V glomerurlonephritis on kidney biopsy (WHO Criteria) (ii) Cerebral Vasculitis, Strokes and Psychosis (iii) Interstitial lung disease with DLCO $<50 \%$ of the predicted value and (iv) Myocarditis, Libmans Sack's verrucous endocarditis etc. One hundred and ten age and sex matched healthy individuals belonging to the same economic status and ethnic background during the same period comprised the controls for this study. These healthy individuals were picked up during population survey.

\section{Determination of Autoantibodies}

Immunofluroscence: All the SLE patients were investigated for anti - nuclear factor (ANF) and antineutrophil cytoplasmic antibodies (ANCA) by indirect immunofluorescence technique using HEP -2 cells as substrate for ANF and human neutrophils from peripheral blood mononuclear cells for anti - neutrophil cytoplasmic antibodies. The results were interpreted in terms of titers, giving fluorescence at the highest dilution of the test sample.
ELISA: The presence of autoantibodies directed towards double stranded DNA (ds DNA), single stranded DNA (ss DNA), ribonucleoprotein (nRNP), Smith antigen (Sm), SS-A/ Ro and SS-B/ La were tested using the commercially available ELISA kits (Clarks Diagnostics USA). Anti histone antibodies to whole molecule was tested by standardizing an ELISA using Histone type II AS (Sigma, USA) using commercial standards (high, medium and low; from Novamed, Israel) (Krippner et al. 1984; Burlingame and Rubin 1990). Rheumatoid factor was detected using commercially available kit (Rashmi diagnostics, India).

HLA Tissue Typing: Ten to fifteen milliliters of venous blood (in heparin $50 \mathrm{IU} / \mathrm{ml}$ ) was collected in a sterile tube from each individual. The lymphocytes were isolated by density gradient centrifugation on Histopaque (Boyum 1968). HLA A, and B locus antigens were identified by NIH two - stage Microlymphocytotoxicity assay (Terasaki and Mc Clleland 1964). The antisera were indigenous

Table 1: Clinical profile among SLE patients from Mumbai

\begin{tabular}{lrr}
\hline Clinical manifestations & $\begin{array}{c}\text { Number } \\
\text { positive }\end{array}$ & Percentage \\
\hline Type of Skin Involved & & \\
Butterfly Rash & 6 & 13 \\
Sclerodermal Facial Rash & 12 & 26 \\
Discoid Rash & 4 & 9 \\
Photosensitivity & 16 & 35 \\
Mild Rash & 4 & 9 \\
Erythematous patches & 3 & 7 \\
Alopecia & 6 & 13 \\
Nature of Joint Involved & & \\
Arthralgia & 3 & 7 \\
Arthritis & 32 & 70 \\
Serositis & & \\
Pleuritis & 7 & 15 \\
Pericarditis & 3 & 7 \\
Haematological & & \\
Immune Haemolytic Anemia & 2 & 4 \\
Immune thrombocytopenia & 2 & 4 \\
Kidney & & \\
Haematuria & 4 & 9 \\
Oedema of Feet & 10 & 22 \\
Renal disorders & 13 & 28 \\
Lupus Nephritis & 2 & 4 \\
CNS & & \\
Psychosis & 2 & 4 \\
Epilepsy & 1 & 2 \\
Miscellaneous & & \\
Facial Oedema & 6 & 2 \\
Cutaneous Vasculitis & 13 \\
Oral Ulcer & 7 & 15 \\
Haematuria & & \\
\hline & &
\end{tabular}


(Shankarkumar et al. 1998) and commercial (Biotest, Germany; Behring, Germany; Pelfreez, USA) in origin.

\section{Statistical Analysis}

The phenotype frequencies, odds ratio, probability value, confidence intervals were estimated using our database and computer programs as described earlier (Shankarkumar et al. 2002). Since each individual is tested for several HLA alleles and the same data used for comparing the frequency; it is possible that one of the alleles will by chance deviate significantly. To overcome this error, the $\mathrm{P}$ value is corrected by use of Bonferoni inequality method (Dunn 1961).

\section{RESULTS}

\section{HLA Association Studies}

The phenotype frequencies of HLA A and B antigens in SLE patients from Mumbai were compared with the controls (Table 2). A Significant increase was observed in the frequency of HLA A1, A2, and B27 among total SLE patients $(\mathrm{A} 1=39.6 \%$ vs $17.8 \% ; \mathrm{B} 27=12.2 \%$ vs $2.3 \%$ ). HLA A19, and B15 were found to be decreased in the patient group compared to the controls (A19 $=26.5 \%$ vs $43.4 \%$; B $15=2.0 \%$ vs

Table 2: HLA association in total SLE patients from Mumbai

\begin{tabular}{lrrrr}
\hline HLA & $\begin{array}{r}\text { Patients } \\
(\% P F) \\
N=53\end{array}$ & $\begin{array}{r}\text { Controls } \\
(\% P F) \\
N=110\end{array}$ & OR & $<P$ Value \\
\hline A1 & 39.60 & 17.80 & 2.99 & 0.001 \\
A2 & 45.30 & 29.70 & 1.95 & 0.001 \\
A3 & 24.50 & 16.80 & 1.61 & \\
A9 & 32.70 & 38.80 & 0.78 & \\
A11 & 12.20 & 21.70 & 0.53 & \\
A19 & 26.50 & 43.40 & 0.48 & 0.001 \\
A26 & 2.00 & 1.60 & 1.58 & \\
A28 & 15.10 & 11.90 & 1.34 & \\
B5 & 28.60 & 27.10 & 1.09 & \\
B7 & 20.40 & 17.80 & 1.20 & \\
B12 & 24.50 & 20.20 & 1.30 & \\
B15 & 2.00 & 16.30 & 0.16 & 0.001 \\
B22 & 10.20 & 6.20 & 1.77 & \\
B27 & 12.20 & 2.30 & 5.40 & 0.001 \\
B35 & 28.60 & 27.10 & 1.09 & \\
B40 & 34.70 & 27.90 & 1.38 & \\
\hline
\end{tabular}

$\% \mathrm{PF}=$ percentage phenotype frequency, $\mathrm{OR}=$ Odds Ratio.
$16.30 \%$ ). However the differences for HLA A10, $\mathrm{B} 18$ and B22 did not remain significant after the $\mathrm{P}$ value correction was applied. It was further interesting to note that among the major organ involved severe patients A3, A28, and B7 where increased while A11, A19 and B17 were decreased (Fig. 1). Among the minor organ involved patients it was A1, A2, A26, B5, B40 and B56 which were increased while A11, A28 and B7 were decreased (Fig. 2).

\section{Autoantibodies Identified}

The table 3 present the autoantibodies that were identified in the Major and Minor organ involved SLE patients studied. All the SLE patients with major organ involvement studied presented with high titer of ANF, AHA and anti -Sm antibodies. It was observed that $54 \%$ of the patients were anti - dsDNA positive, $10 \%$ had anti- Ro/SS-A antibodies, $22 \%$ had anti-La/ SS-B antibodies, $14 \%$ had both Ro SS-A and La SS-B antibodies. It was interesting to observe that out of $54 \%$ anti - ds DNA positive patients $44 \%$ presented skin manifestations, $59 \%$ presented with joint involvement, $26 \%$ presented with pleuritis and/or pericarditis and $7 \%$ had CNS involvement. Further $66 \%$ of the SLE patients had kidney involvement, $46 \%$ had facial or skin rash and/or photosensitivity, $56 \%$ had severe joint involvement with pain and arthritis and $16 \%$ had CNS involvement while only one patient presented with Raynaud's phenomenon.

Table 3: Autoimmune profile of sle patients with major and minor organ involvement

\begin{tabular}{lcc}
\hline Autoantibodies & $\begin{array}{c}\text { Major organs } \\
\text { Percentage }(+v e)\end{array}$ & $\begin{array}{c}\text { Minor organs } \\
\text { Percentage (+ve) }\end{array}$ \\
\hline ANF & 95 & 88 \\
Anti - ds DNA & 53 & 75 \\
Anti - ss DNA & 26 & 38 \\
Anti - Sm & 32 & 25 \\
Anti -nRNP & 26 & 38 \\
Anti - SS-A/ Ro & 16 & 25 \\
Anti - SS-B/ La & 5 & 38 \\
RF & 16 & 19 \\
ANCA & 5 & 6 \\
\hline
\end{tabular}

\section{DISCUSSION}

Autoantibodies are a prominent feature of some autoimmune disorders and the significance of such antibodies could be diagnostic as well as to differentiate the patients into subsets during the course of prognosis. SLE is a classical 


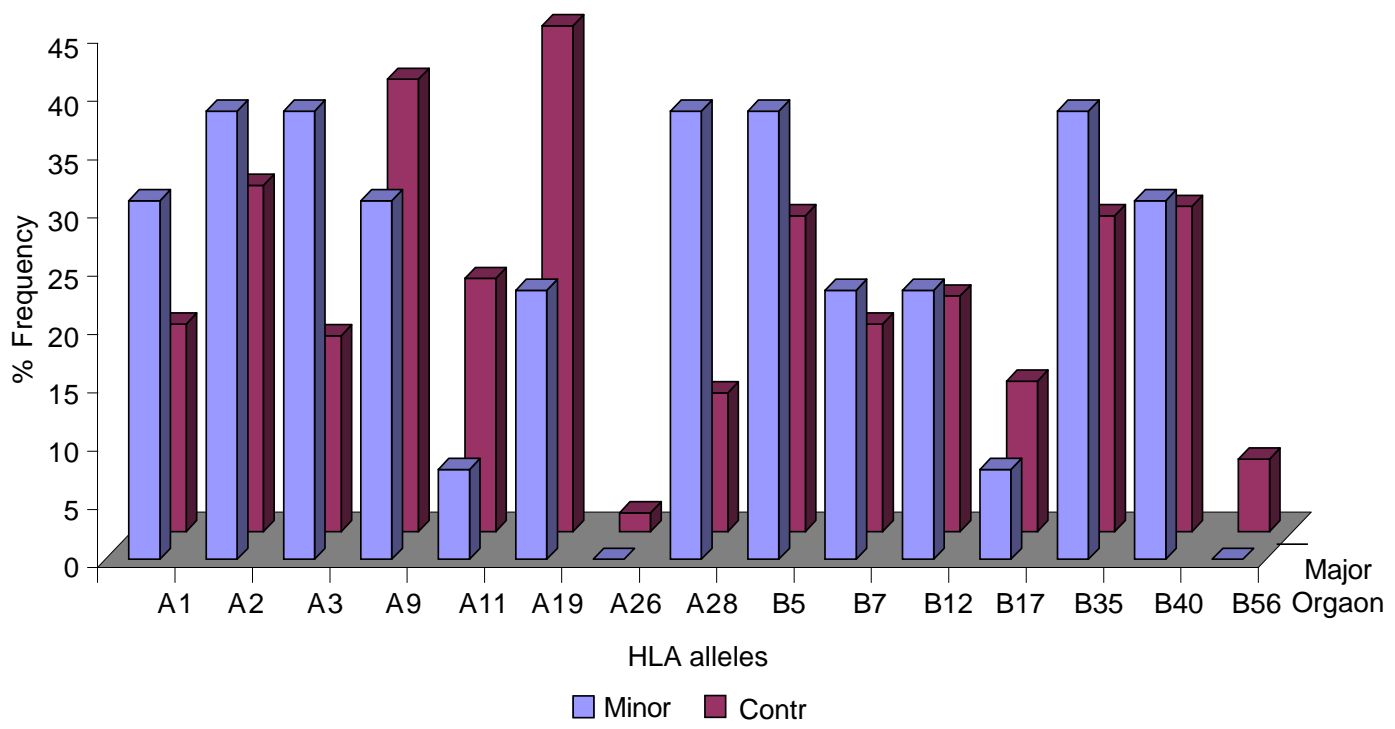

Fig. 1. HLA distribution among the major organ involved SLE patients

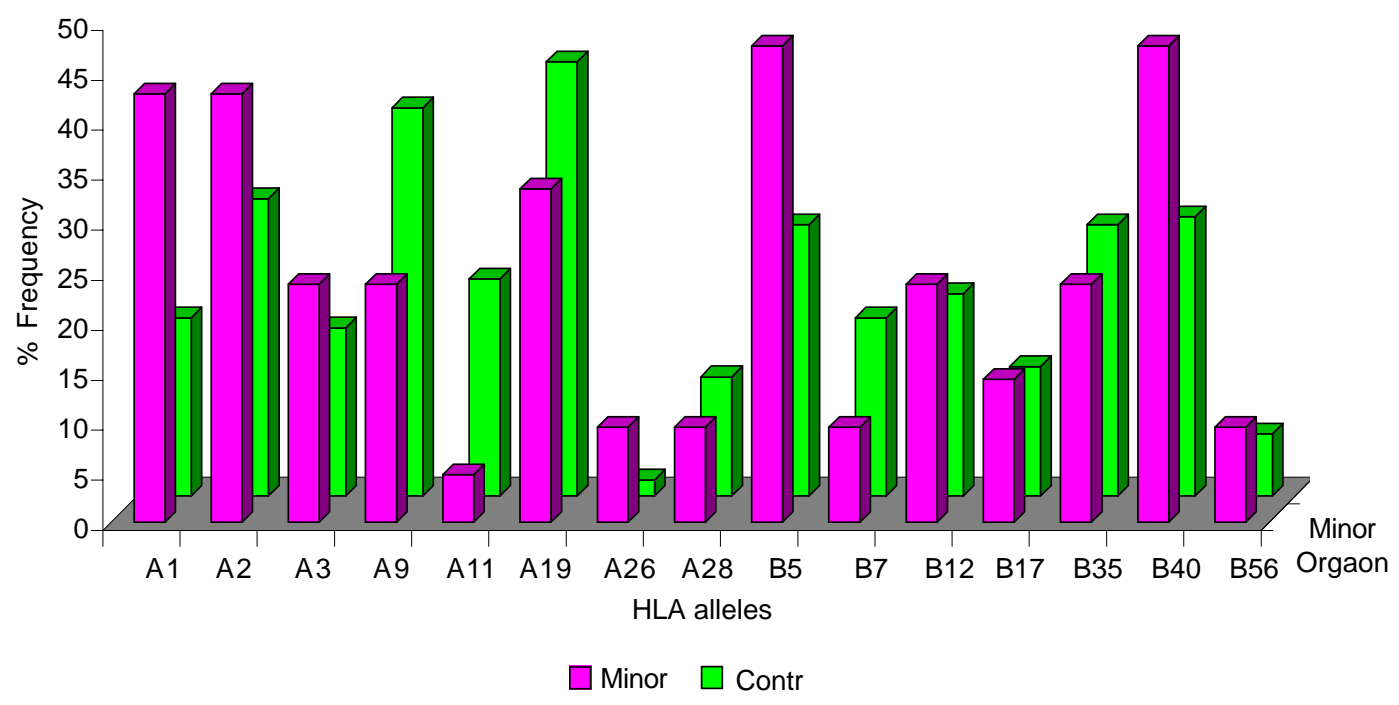

Fig. 2. HLA distribution among minor organ involved SLE patients and controls

autoimmune disease characterized for the presence of antibodies like ANF, anti - ds DNA, anti - Sm, anti - SS-A/ Ro, anti- SS-A/ La etc. Our earlier reports from western India have shown the presence of ANF, anti dsDNA in SLE cases (Chablani et al. 1987; Badakere and Chablani 1994). The positive correlation between increased anti dsDNA antibody levels and the prevalence of anti-SS-A/ Ro has been noticed among SLE patients (Dillon et al. 1983). The relevance of autoantibody anti- SS-B/ La as a marker of distinctive SLE subset has also been reported (Maddison et al. 1998). Further high incidence of anti SS-A/ Ro and anti S-B/ La antibodies have been reported. A few of the SLE subsets are associated with Sm and nRNP antibodies and 
are known to exhibit clinical expression of the disease (Tan et al. 1982). Our results for nRNP and anti dsDNA antibodies in the present study corroborate the results of Notman et al. (1975). Recently a significant association and increased evidence of direct involvement in the pathogenesis of tissue injury by the anti SS-A/ Ro and SS-B/ La antibodies are reported (Mavragani et al. 2000).

In SLE multiple loci within the MHC have been implicated in susceptibility like HLA class II alleles, Complement components and TNF loci (Naves et al. 1998; Gladman et al. 1999; Galeazzi et al. 2000; Huang et al. 2001; Azizah et al. 2001; Shankarkumar et al. 2003). HLA DQ1 and DQ2 are also found to be associated with Ro, La, Sm and dsDNA autoantibodies. HLA DR2 - DQ1 haplotype associated with SLE has low TNF levels (Komata et al. 1999; Rood et al. 2000). A review based on the clinical and laboratory characters in Indian SLE patients showed higher proportion of alopacia, renal lupus, oral ulcers and neurological involvement reaching statistically significant levels when compared to other racial groups (Malaviya et al. 1997) and reduced life expectancy in Indian SLE patients (Murali et al. 1997). However little studies have shown class I allele associations with SLE. HLA studies from SLE patients from North India showed appreciable risk of HLA DR4 among the patients, additionally the haplotype B8-DR4 was frequently encountered in the patient group (Mehra et al. 1993). Recently HLA DRB $1 * 03$ and DQB $1 * 0302$ alleles have been shown to be associated in Western Indian SLE patients (Shankarkumar et al. 2003). In our present study HLA B8 was not found to be associated as in the North Indian SLE patients, while we have observed the A3, A28 and B27 may have association while the A11 and A19 may have a protective role in our SLE patients from Mumbai. Interethnic differences in the associations of TNF promoter polymorphism with SLE have also been reported from UK South Africa, and Iceland (Steinsson et al. 1998).

In summary our result shows a significant twofold increase in the odds ratio for HLA A3, A28 and B27 alleles with increased Ro, La, Sm and ds DNA autoantibodies when compared to controls. Further a significant twofold decrease in the odds ratio for HLA A19, A11, B7 and B15 alleles with decreased Ro, La, Sm and ds DNA autoantibodies, when compared to normal controls suggesting the positive and negative associations of these HLA alleles among the SLE patients from Mumbai. The associations support the importance of ethnic background and indicate that relative importance of different genes may vary in different populations as observed in other reported ethnic populations around the world.

\section{REFERENCES}

Alarcon- Segoria D 1984. The pathogenesis of immune dysregulation in systemic lupus erythematosis. A troika. J Rheumatol, 11: 588-90.

Azizah MR, Ainoi SS, Kong NC, Normaznah Y, Rahim MN 2001. HLA antigens in Malay patients with systemic lupus erythematosus: association with clinical and autoantibody expression. Korean $J$ Intern Med, 16: 123-131.

Badakere SS, Chabalani AN 1994. Significance of antibodies to ENA ( Sm + RNP) and patterns of immunofluorescence in the diagnosis of some collagen vascular autoimmune disorders. Ind $J$ Hematol Blood Tranf, 12: 107-112.

Bale UM, Mehta MM, Contractor NM, Bhatia HM, Tilve GH 1980. HLA antigens in ankylosing spondylitis: the association of HLA B27. Ind $\mathrm{J}$ Med Res, 71: $96-103$.

Benacerraf B 1981. Role of MHC gene products in immune regulation. Science, 212: 1229-38.

Boyum A 1968. Separation of leucocytes from Blood and Bone marrow. Scan J Clin Lab Invest, 21 (Spp): $97-99$.

Burlingame R, Rubin R 1990. Subnucleosome structure as substrates in Enzyme linked immunoabsorbant assays. J Immunol Methods, 134: 187- 99.

Chablani AT, Badakere SS, Bhatia HM 1987. Non organ specific autoantibodies associated with autoimmune neonatalogical disorders and systemic autoimmune disease. Ind J Med Res, 86: 337-342.

Dillon CF, Jones JV, Reichlin M 1983. Autoantibody to Ro in a population of patients with SLE: distribution, clinical and serological associations. J Rheumatol, 10: $380-386$

Dunn OJ 1961. Multiple comparison among means. Am J Stat Assoc, 56: 52 - 56.

Eroglu GE, Kohler PF 2000. Familial Systemic lupus erythematosus. The role of genetic \& environmental factors. Ann Rheum Dis, 61: 2931 .

Galeazzi M, Sebastiani GD, Tincani A et al. 2000. HLA class II alleles associations of anticardiolipin and anti-beta2GPI antibodies in a large series of European patients with systemic lupus erythematosus. Lupus, 9: 47-55.

Gladman DD, Urowitz MB, Darlington GA 1999. Disease expression and class II HLA antigens in systemic lupus erythematosus. Lupus, 8: 466-70.

Huang JL, Shaw CK, Lee A, Lee TD, Chou YH, Kuo ML 2001. HLA DRB1 antigens in Taiwanese patients with juvenile onset systemic lupus erythematosus. Rheumatol Int, 21:103-105.

Komata T. Tsuchiya N, Matsushita M, Hagiwara K, Tokunaga K 1999. Associations of tumor necrosis 
factor receptor 2 (TNFR2) polymorphism with susceptibility to systemic lupus erythematosus. Tissue Antigens, 53: 527-533.

Krippner H, Springer B, Merle S, Pirlet K 1984. Antibodies to Histone of the $\operatorname{IgG}$ and $\operatorname{IgM}$ class in systemic lupus erythematosis. Clin Exp Immunol, 58: $49-56$.

Maddison PJ, Skinner RP, Vlachoyiannopoulos P, Brenaud DM, Hough D 1998. Antibodies to n RNP, Sm, Ro (SSA), La (SSB) detected by ELISA: their specificity and interrelations in connective tissue disease sera. Clin Exp Immunol, 62: 337-345.

Malaviya AN, Chandrasekaran AN, Kumar A, Shamar PN 1997. Systemic lupus erythematosis in India. Lupus, 6: 690-700.

Mavragani CP, Tiofas AG, Moutsopoulouis HM 2000. Sjogerns syndrome: autoantibodies to cellular antigens. Clinical and molecular aspects. Int Arch Allerg Immunol, 123: 46-57.

Mehra NK, Pande I, Taneja V et al. 1993. Major Histocompatibility complex genes and susceptibility to systemic lupus erythematosus in Northern India. Lupus, 2: 313-14.

Murali R, Jeyaseelan L, Rajarathnam S, John L, Ganesh A 1997. Systemic lupus erythematosis in Indian patients. Prognosis, survival and life expectancy. Nat Med J India, 10: 159-164.

Naves M, Hajeev AH, Teh LS et al. 1998. Complement C4B null allele status confers risk for systemic lupus erythematosis in a Spanish population. Eur J Immunogenet, 25: 317-320.

Notman DD, Kerata N, Tan EM 1975. Profiles of anti nuclear antibodies in systemic rheumatic diseases. Ann Intern Med, 83: 464 - 469.

Rood M.J, van Krugten MV, Zanelli E et al. 2000. TNF
- 308A and HLA DR3 alleles contribute independently to susceptibility to systemic lupus erythematosus. Arthritis Rheum, 43: 129 - 134.

Shankarkumar U, Gupte SC, Gupte S, Pednekar SV, Ghosh K, Mohanty D 1998. Frequency and potential application of HLA antibodies from pregnant women in Mumbai. J Bio Science, 23: 601 -604.

Shankarkumar U, Devaraj JP, Ghosh K, Mohanty D 2002. Seronegative spondarthritis and HLA association. Br J Biomed Sci, 59: 38-41.

Shankarkumar U, Ghosh K, Badakere S, Mohanty D 2003. HLA DRB $1 * 03$ and DQB $1 * 0302$ associations in a subset of severely affected SLE patients from western India. Ann Rheum Dis, 62: 92-93.

Shoenfeld Y, Isenberg D 1989. In: JL Turk (Ed.): The Mosaic of Autoimmunity. New York: Elsiever Science Publishers. Pp. 349 - 50.

Steinsson K, Jonsdottir S, Arason GJ et al. 1998. A study of the association of HLA DR,DQ and Complement $\mathrm{C} 4$ alleles in systemic lupus erythematosus in Iceland. Ann Rheum Dis, 58: 503-5.

Tan EM, Cohen AS, Fries JF et al. 1982. The 1982 revised criteria for the classification of systemic lupus erythematosis. Arth Rheum, 25: 1271-77.

Tersaki PI, McClellend JD 1964. Microdroplet assay of human serum cytotoxins. Nature, 204: 998 - 00.

Tiwari JL, Terasaki PI 1985. HLA and Disease associations. New York: Springer Verlag. Pp. 363369.

Tsuchiya N, Kawasaki A, Tsao BP, Komata T, Grossman JM, Tokunaga K 2001. Analysis of the association of HLA DRB1, TNF alpha promoter and TNFR2 (TNFRSF1B) polymorphisms with SLE using transmission disequilibrium test. Genes Immun, 2: 317-22. 